\title{
Disco de secchi, ictiômetro, puçá e tanque-rede como tecnologias sociais na
}

\section{piscicultura}

\author{
Secchi disk, ichthyometer, bowl and net-tank as social technologies in fish farming \\ Disco de Secchi, ictiómetro, cuenco y tanque de red como tecnologías sociales en la piscicultura
}

Recebido: 15/11/2020 | Revisado: 17/11/2020 | Aceito: 18/11/2020 | Publicado: 01/01/2021

\author{
Jéssica Paloma Pinheiro da Silva \\ ORCID: https://orcid.org/0000-0002-5078-3125 \\ Instituto Federal de Educação, Ciência e Tecnologia do Pará, Brasil \\ E-mail: j.palomaatm@gmail.com \\ Fabricio Nilo Lima da Silva \\ ORCID: http://orcid.org/0000-0002-6402-0540 \\ Instituto Federal de Educação, Ciência e Tecnologia do Pará, Brasil \\ E-mail: fabricio.nilo@ifpa.edu.br
}

\begin{abstract}
Resumo
O objetivo deste estudo foi desenvolver tecnologias sociais para piscicultura familiar, que cultivam tambaqui (Colossoma macropomum). As tecnologias foram construídas durante o curso de Boas Práticas de Manejo no município de Curralinho, arquipélago do Marajó (Pará - Brasil), em março de 2020. Ao final do curso, foi conduzida uma oficina para produção dos equipamentos de manejo, entre estudantes, professores e aquicultores locais. O projeto capacitou homens e mulheres de diversas comunidades rurais. O disco de Secchi, ictiometro, puçá e tanque-rede, foram as tecnologias construídas e testadas nas pisciculturas locais. Em conclusão, as atividades de extensão e a construção de equipamentos de baixo custo para piscicultura familiar, foram bem sucedidas. As tecnologias sociais construídas, se mostraram tecnicamente viável, sendo uma alternativa sustentável para produção e manejo de tambaqui local.
\end{abstract}

Palavras-chave: Aquicultura; Tambaqui; Manejo; Oficina; Inovação.

\begin{abstract}
The objective of this study was to develop social technologies for family fish farming, which cultivate tambaqui (Colossoma macropomum). The technologies were built during the course of Good Management Practices in the municipality of Curralinho, Marajó archipelago (Pará - Brazil), in March 2020. At the end of the course, a workshop was held to produce the management equipment, among students, teachers and local fish farmers. The project trained men and women from different rural communities. Secchi's disk, ichthyometer, bowl and net-tank were the technologies built and tested in local fish farms. In conclusion, the extension activities and the construction of low cost equipment for family fish farming were successful. The social technologies built, proved to be technically viable, being a sustainable alternative for the production and management of local tambaqui.
\end{abstract}

Keywords: Aquaculture; Tambaqui; Management; Workshop; Innovation.

\section{Resumen}

El objetivo de este estudio fue desarrollar tecnologías sociales para la piscicultura familiar, que cultivan tambaqui (Colossoma macropomum). Las tecnologías fueron construidas durante el curso de Buenas Prácticas de Gestión en el municipio de Curralinho, archipiélago de Marajó (Pará - Brasil), en marzo de 2020. Al finalizar el curso, se realizó un taller de producción del equipo de manejo, entre estudiantes, profesores y piscicultores locales. El proyecto capacitó a hombres y mujeres de diferentes comunidades rurales. El disco, el ictiómetro, el tazón y el tanque de red de Secchi fueron las tecnologías construidas y probadas en las piscifactorías locales. En conclusión, las actividades de extensión y la construcción de equipos de bajo costo para la piscicultura familiar fueron exitosas. Las tecnologías sociales construidas demostraron ser técnicamente viables, siendo una alternativa sustentable para la producción y manejo del tambaqui local.

Palabras clave: Acuicultura; Tambaqui; Gestión; Taller; Innovación.

\section{Introdução}

O Brasil apresenta um enorme potencial para o desenvolvimento da aquicultura (Silva et al., 2018; Medeiros et al., 2017). Os recursos hídricos da Amazônia, o clima favorável e à condição geográfica, são fatores essenciais para o crescimento da mesma (Dantas et al., 2019; Ferreira et al., 2020). A criação de peixes no Estado do Pará se encontra em crescimento, sendo 
uma atividade desenvolvida por aquicultores(as) de pequeno, médio e grande porte. Uma atividade também exercida pela agricultura familiar, extrativistas e ribeirinhos (Aizawa et al., 2014; Zacardi et al., 2017), com perspectiva de crescimento no arquipélago do Marajó.

Os aquicultores no Marajó, por exemplo, cultivam o tambaqui (Colossoma macropomum Cuvier, 1818), para a subsistência e, o excedente comercializam. Porém, a cultura da espécie é praticada em estruturas de pequeno porte, com baixo desenvolvimento tecnológico e utilizam conhecimento empírico. A piscicultura local contribui para o desenvolvimento rural, possibilita o retorno econômico aos produtores, colabora para a geração de emprego e otimiza os recursos naturais existentes nas propriedades. De forma contrária, muitos produtores vem apresentando problemas ao desenvolver a atividade, por falta de Boas Práticas de Manejo (BPM) na piscicultura. Uma alternativa consiste em desenvolver tecnologias sociais que sejam adaptáveis aos problemas vivenciados nessa região da Amazônia.

Tecnologia social envolve todo o produto, método, processo ou técnica que são criados juntamente com a comunidade para solucionar algum tipo de problema social (Dagnino, 2011). Essas tecnologias, devem atender os quesitos de simplicidade, baixo custo, fácil aplicabilidade e impacto social comprovado na piscicultura (Medeiros et al., 2018). Segundo Oliveira et al. (2012), ainda há muitos desafios no que se diz respeito a transferência de tecnologia. Diante do exposto, o objetivo do apresente trabalho de extensão foi desenvolver quatro tecnologias sociais para piscicultura de caráter familiar no Marajó.

\section{Metodologia}

O trabalho foi executado no município de Curralinho, arquipélago do Marajó (Pará - Brasil), em março de 2020 (Figura 1). O Marajó está localizado na costa amazônica (Amaral et al., 2012). Região que compreende 16 municípios, os quais compõem as microrregiões de Arari (Cachoeira do Arari, Chaves, Muaná, Ponta de Pedras, Salvaterra, Santa Cruz do Arari e Soure), Furos de Breves (Afuá, Anajás, Breves, Curralinho e São Sebastião da Boa Vista) e Portel (Bagre, Gurupá, Melgaço e Portel). Curralinho possui uma extensão territorial de $3.620,279 \mathrm{~km}^{2}$, sua população é estimada em 33.893 pessoas para 2018 (IBGE, 2010). A maioria dessa população é ribeirinha, devido à cidade ser cercada por rios e ilhas, são aquicultores, pescadores, extrativistas e agricultores familiares.

O projeto, foi conduzido por estudantes regularmente matriculados no curso de Licenciatura em Educação do Campo e do curso Técnico em Aquicultura do Instituto Federal de Educação, Ciência e Tecnologia do Pará (IFPA), Campus Breves. Eles participaram de todas as etapas do projeto, atuando nas condições de pesquisadores e instrutores, construíram as tecnologias sociais para cultura do tambaqui, dentro do curso de capacitação em Boas Práticas de Manejo (BPM), conforme procedimentos de ação empregadas por Köche (2016). Utilizou-se da metodologia ativa, tornando os envolvidos agentes principais de sua aprendizagem (Pereira, et al., 2018). Para isso, professores, estudantes e cursistas participaram da oficina de construção. Foi adotada uma linguagem simples durante as construções, a fim de proporcionar um melhor entendimento por parte dos piscicultores. Foram utilizados materiais alternativos e de baixo custo, levando em consideração a realidade da piscicultura local. 
Figura 1 - Localização do município de Curralinho, arquipélago do Marajó, Pará, Brasil.
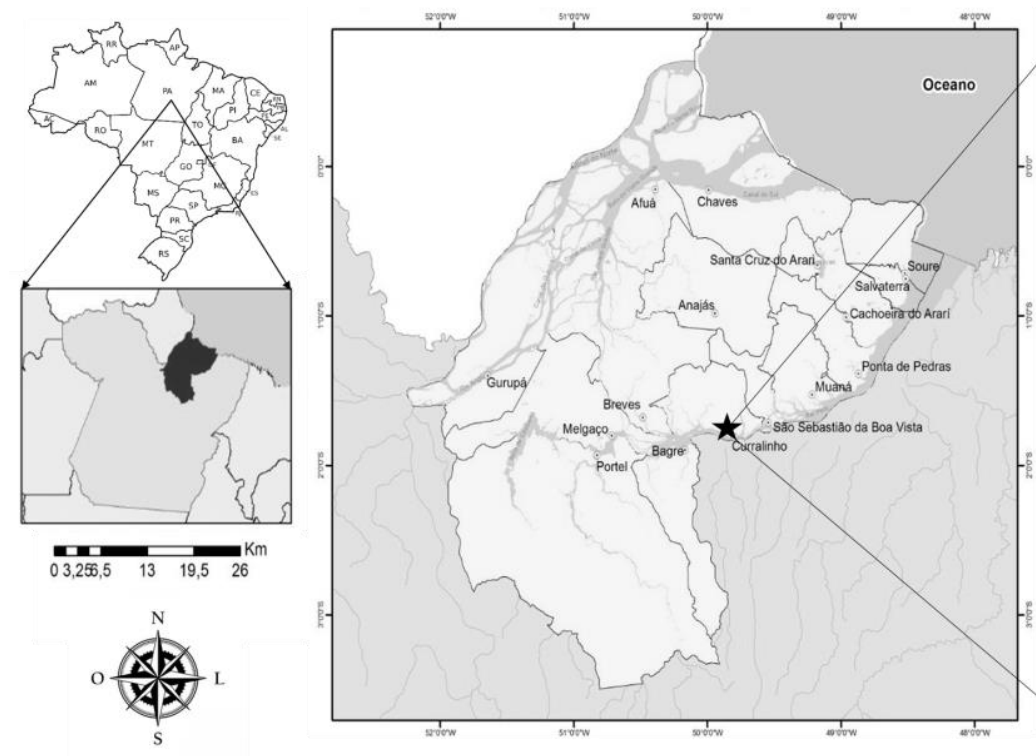

Fonte: Elaborado por Christian Nunes.

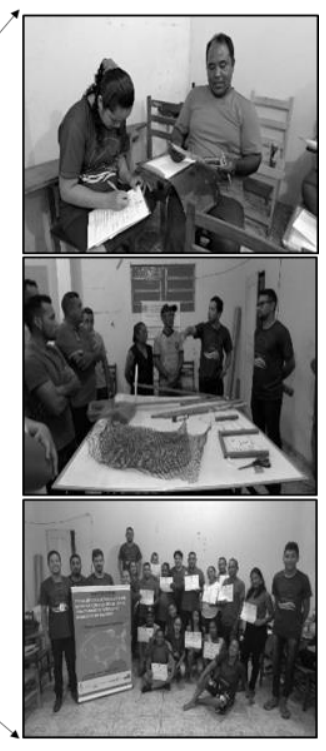




\section{Confecção do tanque-rede}

Com a função de reter determinado número de indivíduos, permitindo o livre fluxo de água, o tanque-rede foi confeccionado utilizando os seguintes materiais: madeira (podendo ser cabo de vassoura), tela de polietileno (reaproveitável), pregos e martelo. O primeiro passo consistiu no corte da madeira, nas seguintes dimensões: quatro pedaços de $50 \mathrm{~cm}$ e oito pedaço de $30 \mathrm{~cm}$. Após o corte, as partes foram unidas, com auxílio de prego e martelo, em seguida com amarras foi fixada a tela, observando para que ficasse bem presa.

\section{Resultados e Discussão}

A Figura 2, apresenta as tecnologias construídas para piscicultura familiar. A difusão e a transferência são partes indissociáveis do mesmo processo, que inclui a geração de tecnologia (Farias \& Mendes, 2009). Esse processo tem como finalidade a adoção de inovações pelos aquicultores, visando o desenvolvimento sustentável.

No processo produtivo, analisar os parâmetros físicos e químicos da água, permite ao piscicultor controlar e adequar o ambiente de suas criações, promovendo bom desenvolvimento dos organismos aquáticos, tornando a atividade economicamente viável (Leira et al., 2017). Condições inadequadas da qualidade da água comprometem o sucesso dos sistemas de aquicultura, acarretando em problemas no cultivo e até mesmo a sobrevivência dos peixes (Lachi \& Sipaúba, 2018). 
Figura 2 - Tecnologias sociais: A) disco de Secchi, B) ictiômetro, C) puçá e D) tanque-rede, desenvolvidos para piscicultura familiar.

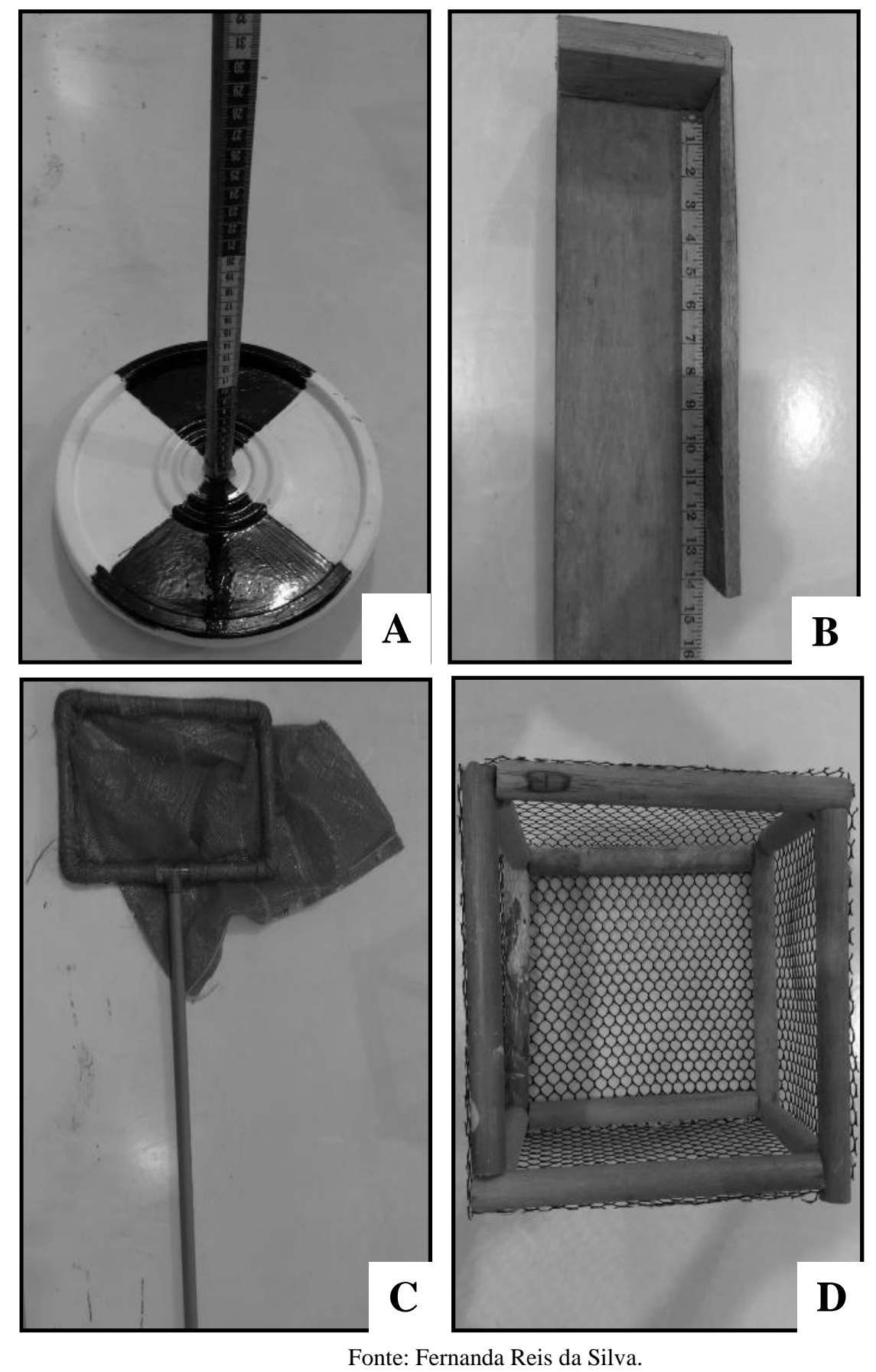

Dentre os parâmetros físicos que influenciam na qualidade da água, está a transparência (cor, turbidez e sólidos). Esta variável refere-se a medida da profundidade, em centímetros, da capacidade de penetração da luz na água, que varia com a presença de fitoplâncton e/ou excesso de suspensão de sedimentos finos (silte e argilas) (Lopes, 2012). A leitura da transparência é feita com um equipamento denominado disco de Secchi. Dada a sua importância de uso na piscicultura. O disco de Secchi foi a primeira tecnologia social confeccionada para piscicultura familiar.

Para realizar a aferição da transparência, o piscicultor deve mergulhar o disco de Secchi lentamente na água, em posição vertical até que não seja possível enxergá-lo. Quando este limite é identificado, marca-se na fita graduada a distância de transparência em centímetro e, em seguida, realiza-se o registro dos dados (Matos et al., 2016). É importante, que 
juntamente com essa informação, insira também informações sobre a cor da água (verde - presença de fitoplâncton, barrenta excesso de argila) (SENAR, 2019).

Recomenda-se que o monitoramento da qualidade da água através do uso do disco de Secchi, seja realizado a cada semana ou a cada três dias, preferencialmente em dias de sol e aproximadamente no mesmo horário. A transparência mais adequada para o sistema de piscicultura está entre 30 e $60 \mathrm{~cm}$ de profundidade, indicando uma densidade apropriada de fitoplâncton. Medidas acima de $60 \mathrm{~cm}$ indica a possibilidade de invasão de macrófitas, e menor que $30 \mathrm{~cm}$ indicativo de riscos iminentes com o oxigênio dissolvido e variação no pH, devendo realizar manejo adequado (Lopes, 2012; SENAR, 2019).

O acompanhamento do desenvolvimento dos peixes utilizando a biometria é uma etapa importante do processo de manejo durante o cultivo. A biometria consiste na coleta e análise de amostras e informações que auxiliam no ajuste do manejo da produção, principalmente no que se refere a alimentação (Lima et al., 2013).

O manejo da biometria envolve a captura, medição do tamanho e pesagem de uma amostra de peixes do viveiro, que seja representativa. Torna-se importante frisar, que os procedimentos realizados fora da água (pesagem e medição), devem ser em menor tempo possível para que os animais sejam devolvidos o mais rápido para o viveiro (Faria et al., 2013) e de forma cuidadosa de maneira a reduzir o estresse, ferimentos e morte dos peixes (Vidal et al., 2008).

A biometria deve ser realizada periodicamente, ao menos uma vez no mês (Tavares-Dias \& Fujimoto, 2014), preferencialmente após jejum de $24 \mathrm{~h}$ e nas primeiras horas do dia. Dentre os materiais comumente utilizados para a prática da biometria na piscicultura estão a rede de captura; ictiometro; puçás; balança; baldes. Nesse contexto, os instrumentos de ictiometro, puçá e tanque-rede, foram as tecnologias sociais confeccionadas no curso de BPM.

\section{Considerações Finais}

As atividades de extensão e a construção das tecnologias sociais para piscicultura familiar foram bem sucedidas. O disco de Secchi, ictiômetro, puçá e o tanque-rede, se mostraram tecnicamente viável, sendo uma alternativa sustentável e economicamente viável para produção e manejo de tambaqui local. O protagonismo dos piscicultores para elaboração das tecnologias sociais contribuiu para adequação do produto. A autonomia deste na participação e apropriação das soluções sustentáveis efetivas, são fundamentais para a replicação da tecnologia, sendo este o mecanismo chave para o sucesso do projeto.

Por fim, espera-se que este projeto sirva de subsídio demonstrativo da importância da elaboração e aplicação de tecnologias sociais para o meio rural, como instrumento de alcance de melhoria nos âmbitos econômicos e produtivos, e sirva como fonte de consulta e auxílio para replicação das tecnologias desenvolvidas. Sugerimos a idealização de novas tecnologias sociais que apreciem as problemáticas existentes na operabilidade da produção desses aquicultores.

\section{Agradecimentos}

À Pró-Reitoria de Extensão (PROEX) do Instituto Federal de Educação, Ciência e Tecnologia do Pará (IFPA), pelo financiamento na pesquisa (Edital nº 03/2019). À Prefeitura municipal de Curralinho e a Colônia de Pescadores Z-37, pelo apoio estrutural durante o projeto. Aos estudantes bolsistas e voluntários, envolvidos no projeto "Piscicultura Marajoara" do IFPA Campus Breves.

\section{Referências}

Aizawa, N., \& Masuda, M., Ito, L. S. (2014). Current situation of freshwater aquaculture in the lower Amazon River and the potentiality of development. Tropics, 23(3), 127-134. 
Amaral, D. D., Mantelli, L. R, \& Rossetti, D. F. (2012). Paleoenvironmental control on modern forest composition of southwestern Marajo Island, Eastern Amazonia. Water and Environment Journal, 26(1), 70-84.

Dantas, D. P., Flickinger, D. L., Costa, G. A., Batlouni, S. R., Moraes-Valenti, P., \& Valenti, W. C. (2019). Technical feasibility of integrating Amazon river prawn culture during the first phase of tambaqui grow-out in stagnant ponds, using nutrient-rich water. Aquaculture, $516,734611$.

Dagnino, R. (2011). Tecnologia social: base conceitual. Ciência \& Tecnologia Social, 1(1), 1-12.

Farias, J. L. S., Mendes, M. E. P. (2009). Estratégia de transferência de tecnologia como forma de apropriação do conhecimento: o caso Coomanta Sobral. Série Documentos Embrapa Caprinos e Ovinos.

Faria, R. H. S., Morais, M., Souza Soranna, M. R. G., \& Sallum, W. B. (2013). Manual de criação de peixes em viveiros. Brasília: Codevasf.

Ferreira, G., Marcovitch, J., \& Val, A. (2020) "A systematic review of the production chain of the Arapaima gigas, the giant fish of the Amazon". Management of Environmental Quality: An International Journal, 31(2), 349-363.

IBGE - Instituto Brasileiro de Geografia e Estatística. Censo demográfico brasileiro 2010. http://www.ibge.gov.br/home/. IDHM. Atlas do Desenvolvimento.

Köche, J. C. (2016). Fundamentos de metodologia científica. Editora Vozes.

Lachi, G. B., Sipaúba-Tavares, L. H. (2018). Qualidade da água e composição fitoplanctônica de um viveiro de piscicultura utilizado para fins de pesca esportiva e irrigação. Boletim do Instituto de Pesca, 34(1), 29-38.

Leira, M. H., Cunha, L. T., Braz, M. S., Melo, C. C. V., Botelho, H. A., \& Reghim, L. S. (2017). Qualidade da água e seu uso em pisciculturas. PUBVET, 11(1), 11-17.

Lima, A., Silva, A. P. Da, Rodrigues, A., Bergamin, G., Torati, L., Pedroza Filho, M. X., \& Maciel, P. (2013) Biometria de peixes: piscicultura familiar. Embrapa Pesca e Aquicultura-Fôlder/Folheto/Cartilha (INFOTECA-E). Divinópolis, TO: Embrapa Pesca e Aquicultura.

Lopes, J. C. O. (2018). Piscicultura. Lopes-Floriano: EDUFPI, 2012.

Matos, F. T. De, Webber, D., Fontoura, A., Pinho, E., Roubach, R., Bueno, G., \& Barros, D. (2016). Monitoramento de qualidade de água das atividades aquícolas em reservatórios continentais brasileiros. Embrapa Pesca e Aquicultura-Documentos (INFOTECA-E). Palmas, TO: Embrapa Pesca e Aquicultura.

Medeiros, M., Aubin, J., \& Camargo, A. (2017). Life cycle assessment of fish and prawn production: Comparison of monoculture and polyculture freshwater systems in Brazil. Journal od of Cleaner Production, 156, 528-537.

Medeiros, P. A., Oliveira, A. B. De, \& Costa, J. M. (2018). Tecnologias de baixo custo para piscicultura familiar no amazonas, brasil. Nexus-Revista de Extensão do IFAM, 4(1), 11-18.

Oliveira, A. M., Val, V. M. F. A., \& Val, A. L. (2012). Caracterização da atividade de piscicultura nas mesorregiões do estado do Amazonas, Amazônia brasileira. Revista Colombiana de Ciência Animal Recia, 4, 154-162.

Pereira, A. S., Shitsuka, D. M., Parreira, F. B., \& Shitsuka, R. (2018). Metodologia da pesquisa científica. Santa Maria. Ed. UAB/NTE/UFSM. https://repositorio.ufsm.br/bitstream/handle/1/15824/Lic_Computacao_MetodologiaPesquisa-Cientifica.pdf.

SENAR. Serviço Nacional de Aprendizagem Rural. (2019). Piscicultura: manejo da qualidade da água. Brasília: Senar (Coleção Senar, 262).

Silva, L. J. S., Pinheiro, J. O. C., Crescêncio, R., Carneiro, E. F., Pereira, B. P., \& Brito, V. F. S. (2018). Tecnologia e desenvolvimento rural: aspectos do cultivo de tambaqui no município de Rio Preto da Eva, AM. Revista Terceira Margem Amazônia, 3(10), $170-196$. 
Research, Society and Development, v. 10, n. 1, e0210110255, 2021

(CC BY 4.0) | ISSN 2525-3409 | DOI: http://dx.doi.org/10.33448/rsd-v10i1.10255

Tavares-Dias, M., \& Fujimoto, R. (2014). Recomendações para melhorias do manejo sanitário em pisciculturas do Estado do Amapá. Embrapa AmapáComunicado Técnico (INFOTECA-E). Macapá - AP: Embrapa Amapá. 4p.

Vidal, L. V. O., Albinati, R. C. B., Albinati, A. C. L., Lira, A. D. De, Almeida, T. R., \& Santos, G. B. (2008). Eugenol como anestésico para a tilapiado-nilo. Pesquisa Agropecuária Brasileira, 43, 1069-1074.

Zacardi, D. M., Lima, M. A. S., Nascimento, M. M., \& Zanetti, C. R. M. (2017). Caracterização socioeconômica e produtiva da aquicultura desenvolvida em Santarém, Pará. Acta of Fisheries and Aquatic Resources, 5(3), 102-112. 